\title{
A fire spread index for grassland fuels
}

\author{
$\underline{\text { J.J. Sharples }}^{a}$, R.H.D. McRae ${ }^{b}$ \\ ${ }^{a}$ Applied and Industrial Mathematics Research Group, School of physical, Environmental and Mathematical \\ Sciences, University of New South Wales Canberra, Australia. \\ ${ }^{b}$ Australian Capital Territory Emergency Services Agency, Majura ACT, Australia. \\ Email: j.sharples@adfa.edu.au
}

Abstract: Fires in grasslands occur throughout the temperate parts of the globe and in some cases, such as was seen in the 2005 Eyre Peninsula (Wangary) fires, can have significant impacts. To address these issues a number of different models for predicting the rate of spread of fire in grasslands have been developed. In this paper we introduce and discuss a novel index, which incorporates fire weather information and relates to the rate of fire spread in grassland fuels. This so-called spread index has the following form:

$$
S_{\alpha, \mu}(U, F M I)=\frac{\alpha \max (1, U)}{F M I+\mu},
$$

where $U$ is the wind speed $\left(\mathrm{km} \mathrm{h}^{-1}\right)$ and $F M I$ is the fuel moisture index, defined by temperature $T\left({ }^{\circ} \mathrm{C}\right)$ and relative humidity $H(\%)$ as:

$$
F M I=10-0.25(T-H) .
$$

The spread index model has two parameters, $\alpha$ and $\mu$. The parameter $\alpha$ serves as a calibration constant that transforms values of the spread index into values that can be interpreted as rates of spread (in $\mathrm{km} \mathrm{h}^{-1}$ ), whereas the parameter $\mu$ acts to temper the growth of the spread index as $F M I \rightarrow 0$, and can be thought of as representing an intensity dependent indraft wind that counters the prevailing winds at the head of the fire. It is found that the values $\alpha=2.4$ and $\mu=6$ provide for reasonable overall performance.

The performance of the index in predicting rate of spread was evaluated through the use of three datasets. The first of these comprises data recorded at Port Lincoln Airport (SA) surrounding the disastrous Wangary Fire (10-11 January 2005). The second is more representative of moderate summer conditions and is comprised of data recorded at Canberra Airport (ACT) in January 2007. The third dataset comprises information relating to twenty significant historical wildfires. These datasets were used as the basis for comparison of the spread index with an established model for grassfire rate of spread (the CSIRO Grassland Fire Spread model).

The fire spread index was found to deliver rate of spread predictions that are practically equivalent to those derived from the established model. On average the differences in rate of spread predictions derived from the spread index and the CSIRO model were $0.2-0.3 \mathrm{~km} \mathrm{~h}^{-1}$, with the biggest differences encountered during the most extreme fire weather associated with the Wangary fire. While neither model did a particularly good job of reproducing the estimated rates of spread of the historical wildfires, in the case of the most extreme fire behaviour during the Wangary fires, the spread index was found to produce the better rate of spread estimates. Overall, given the vagaries of estimating field values for rate of spread and the lack of experimental data for fires burning under extreme conditions, the simple, linearly-structured spread index was found to give reasonable estimates of rate of spread. Indeed, such estimates were not substantially different to those delivered by the nonlinear CSIRO rate of spread model.

These results have implications for the parsimony of fire behaviour models; in particular they suggest a number of conceptual and pedagogical simplifications that could be made with only minimal changes in model performance. The spread index is also briefly considered in the context of a possible unification of fire spread models across different fuel types.

Keywords: $\quad$ Grassland fire spread, fire weather, wildfire 


\section{INTRODUCTION}

It is well known that meteorological variables have a profound effect on the propagation of wildfires. Specifically, air temperature, atmospheric moisture and wind strength and direction are recognised as having the most direct influence on fire spread. There exist a number of modelling approaches that have incorporated these variables as predictors of fuel moisture content, fire danger rating, rate of fire spread and fire intensity (Viney, 1991; Sharples et al., 2009a; 2009b; Sullivan, 2009).

Relative humidity and temperature, the latter primarily in its role as a proxy for solar radiation, affect the vapour exchange processes that ultimately determine the moisture content of wildland fuels. Moisture present in fuels will absorb heat that would otherwise be available to promote the combustion reaction and so, as a consequence, fuels with lower moisture contents will burn more readily and with greater intensity. The overall effect is for fire to spread more rapidly through fuels with lower moisture content.

The effects of wind are also likely to have an influence on vapour exchange processes but these are not typically accounted for in modelling endeavours (the Canadian Fire Weather Index System is an exception). The more dominant role of wind speed and direction in determining fire spread is due to advection of heat, flames and embers, and the downwind extension of radiative preheating associated with flame tilting.

The present paper introduces and considers an index that combines information on wind speed, temperature and relative humidity to provide a measure of the rate of spread of grassfires. The index embodies the intuitive notion that fires will spread faster as wind strength increases and as fuel moisture decreases. Fuel moisture is specifically described by combining temperature and relative humidity to form a fuel moisture index, which was introduced and discussed by Sharples et al. (2009a), after Pook and Gill (1993). The fuel moisture index has also been shown to compare favourably with predictions from more sophisticated models for fuel moisture content in the absence of free moisture (Sharples and McRae, 2010; Slijepcevic et al., 2013). Since we are interested in bushfire applications, the effects of free moisture are not treated in the present study.

The performance of the fire spread index is assessed through comparison of its rate of spread predictions with those of the model of Cheney et al. (1998) and wildfire rate of spread observations.

\section{AN INDEX FOR GRASSFIRE RATE OF SPREAD}

The index for assessing the moisture content of fine dead fuels, the 'fuel moisture index', FMI (Sharples et al. 2009a), is defined as:

$$
F M I=10-0.25(T-H),
$$

where $T$ is the air temperature in ${ }^{\circ} \mathrm{C}$ and $H$ is the relative humidity in \%. At first glance it would appear that the definition of $F M I$ is not dimensionally correct, since $T$ and $H$ have different units. However, this apparent oversight is overcome by simply noting that the coefficient 0.25 seen in equation (1) has units of ${ }^{\circ} \mathrm{C}^{-1}$ when multiplying $T$ and units of $\%^{-1}$ when multiplying $H$. The form of equation (1) is therefore not strictly correct but is more convenient in terms of notation and has certain pedagogical advantages. FMI is in fact a dimensionless index and should not be considered as giving a direct estimate of fuel moisture content. However, it has been shown that FMI is remarkably effective at emulating the predictions of a number of models for fuel moisture content (Sharples et al., 2009a).

Sharples et al. (2009b) used $F M I$ with wind speed $U\left(\mathrm{~km} \mathrm{~h}^{-1}\right)$, to define an effective new, simpler, fire danger index which compared favourably with current compilations. It was:

$$
F(U, F M I)=\frac{\max (1, U)}{F M I} .
$$

Historically, the notion of fire danger rating has been closely linked with rate of spread. Indeed, in the McArthur Fire Danger Rating systems used in southeastern Australia the rate of spread is taken as directly proportional to fire danger rating (McArthur, 1967). These remarks prompt the consideration of a twoparameter family of functions $S_{\alpha, \mu}$ of wind speed $U\left(\mathrm{~km} \mathrm{~h}^{-1}\right)$ and fuel moisture index $F M I$ defined by

$$
S_{\alpha, \mu}(U, F M I)=\frac{\alpha \max (1, U)}{F M I+\mu} .
$$

Note that the function $\max (1, U)$ returns the maximum value of 1 and the wind speed $U$, and so the numerator in equation (3) has a minimum value of $\alpha>0$ to account for the non-zero rates of fire spread that 
are observed even in the absence of wind. In most cases of interest (i.e. when $U \geq 1$ ) the numerator will simply be $\alpha U$.

Once appropriate values of the model parameters $\alpha$ and $\mu$ have been determined, we will refer to the function $S_{\alpha, \mu}$ as the spread index. The parameter $\alpha$ has the units of rate of spread $\left(\mathrm{km} \mathrm{h}^{-1}\right)$, and is included as a scale factor, or calibration constant, which brings values of the spread index into approximate concordance with rate of spread values. The dimensionless parameter $\mu$ has the effect of tempering the growth of $S_{\alpha, \mu}$ as $F M I$ approaches zero, which will occur whenever the difference between temperature and relative humidity approaches 40 . There is also a physical rationale for introducing $\mu$ in the denominator of the spread index. Indeed, taking rate of spread as proportional to fire line intensity, as the rate of spread increases the fire front will produce a stronger convection column that affects the prevailing wind. It particular, entrainment into the convection column ahead of the fire front will produce an indraft wind that counters the prevailing wind. Hence, under this assumption it is more appropriate to consider a wind speed at the fire front of $U-c R$, where $c$ is the (possibly variable) factor relating rate of spread to indraft strength. Supposing that the actual rate of spread is proportional to the simple fire danger index (2), and assuming that $U-c R \geq 1$, yields

$$
R \approx a \frac{(U-c R)}{F M I},
$$

which upon rearrangement may be written

$$
R \approx a \frac{U}{F M I+\mu}
$$

with $\mu=a c$. Hence the inclusion of $\mu$ in the denominator of equation (3) is consistent with an indraft wind at the fire front that counters the prevailing wind and whose strength varies with the rate of spread of the fire. The parameter $\mu$ will therefore be referred to as the indraft factor. The inclusion of an indraft effect is consistent with other models in use (e.g. Cheney et al., 1998).

\section{DATA AND METHODS}

In this section the data and methods used to evaluate the spread index are introduced and discussed.

\subsection{Fire weather and rate of spread data}

The utility of the spread index is evaluated using three datasets. The first dataset is comprised of 201 halfhourly observations of temperature $\left({ }^{\circ} \mathrm{C}\right)$, relative humidity $(\%)$, wind speed $\left(\mathrm{km} \mathrm{h}^{-1}\right)$ and degree of grass curing $(\%)$ as recorded by the automatic weather station at Port Lincoln Airport (Latitude $-34.60^{\circ}$, Longitude $135.88^{\circ}$, Height $8.5 \mathrm{~m}$ ) between 00:00LT, 9 January 2005 and 00:00LT, 13 January 2005. Grass curing was at its maximum of $100 \%$ throughout this period. This dataset is chosen to be representative of extreme fire weather conditions and covers the period during which the devastating Eyre Peninsula (Wangary) fire burnt.

The second dataset was chosen to be representative of more moderate summer conditions and is comprised of 1001 half-hourly observations of temperature, relative humidity and wind speed as recorded by the automatic weather station at Canberra Airport (Latitude $-35.31^{\circ}$, Longitude $149.20^{\circ}$, Height $577.1 \mathrm{~m}$ ) between 12:30LT, 2 January 2007 and 08:30LT, 23 January 2007. Grass curing values were not known and were assumed to be $100 \%$ in all cases.

The third dataset contains 22 instances of fire weather, degree of grass curing, pasture type and estimated rate of spread data relating to twenty significant historical wildfires. All of the curing values except for one are $100 \%$; the exception was $80 \%$. These data are provided in Cheney et al. (1998, Table 1), but were only discussed in a qualitative sense therein. In the following these data are the subject of a more quantitative comparison between observed and predicted rates of spread.

\subsection{CSIRO grassland fire spread model}

The CSIRO grassland fire spread model (Cheney et al., 1998) is employed as a benchmark against which to compare the performance of the spread index. Cheney et al. (1998) derive two separate rate of spread models for grassland fuels: one for natural pastures and one for pastures that have been cut short or grazed. However, if $R_{n}$ is used to denote the rate of spread predictions arising from the model for natural pastures and $R_{c g}$ is used to denote the rate of spread predictions arising from the cut or grazed pasture model, then based on the 1001 data points in the abovementioned Canberra dataset it can be shown that 


$$
R_{n}=1.18 R_{c g}
$$

with an $R^{2}$ value of 0.99995 . Equation (4) is consistent with the assertion of Cheney and Sullivan (1997, p24) that the rate of spread of a fire burning in natural pastures is about $18-20 \%$ higher than the rate of spread in cut or grazed pastures. Given the simple, linear relationship between the two pasture models it is only really necessary to consider one of them in the following analyses. The model for cut or grazed pastures is chosen for this purpose. The model is written as a product of three separate functions:

$$
R_{c g}=W(U) M(m, U) f(C) .
$$

These functions are $W(U)$, which accounts for the response of rate of spread to wind speed $U, M(m, U)$, which accounts for the response of rate of spread to changes in fuel moisture content $m$ under different wind speed regimes, and $f(C)$, which accounts for the response of rate of spread to changes in grass curing levels. The constituent functions are given by Cheney et al. (1998).

In many situations of interest the fuel moisture measurements required to implement (5) are not available. More commonly available (or at least readily obtainable) are measurements of temperature and relative humidity. Thus to be able to implement the rate of spread model (5) when only temperature and relative humidity data are available the following model for fuel moisture is used:

$$
m(T, H)=9.668-0.207 T+0.137 H .
$$

Equation (6) is an approximate mathematical expression derived from the nomograms of Cheney and Sullivan (1997, p 42 and 44). We note the similarity between $m(T, H)$ and $F M I$.

\subsection{Determination of the scale factor $\alpha$}

For a given value of the parameter $\mu=\mu^{*}$, the parameter $\alpha$ is chosen so that the mean of the spread index values $S_{\alpha, \mu^{*}}$ is equal to the mean of the rate of spread predictions derived from the model (5) of Cheney et al. We note that other methods, such as matching the median values, could have alternatively been used. The main point is that the parameter $\alpha$ determines a function for transforming between the two scales relating to the spread index (3) and the predictions of (5). We note further that the change in scale, represented by $\alpha$, is immaterial if the two scales corresponding to (3) and (5) provide roughly equivalent measures of rate of spread.

\subsection{Determination of the indraft factor $\mu$}

There are good physical and analytical reasons to maintain that the indraft factor $\mu$ varies as the rate of spread varies. For example, Nelson (2003) calculated that the mean entrainment velocity of air drawn into the base of a convection column by fire-induced buoyancy is dependent upon the horizontal velocity of the prevailing wind and the fire line intensity. Moreover, preliminary analyses of model predictions arising from (3) and (5) revealed that the optimal correlation was achieved by taking different values of the indraft factor $\mu$ depending on the range of the rate of spread values considered. However, considering the spread index with a single fixed value of $\mu$ is far more convenient in terms of its conceptualisation and implementation. An optimal value of $\mu$ was therefore sought to yield a spread index that compares favourably with predictions arising from (5) over a broad range of spread rates.

To determine appropriate values of the model parameters $\alpha$ and $\mu$ the Canberra and Port Lincoln datasets were each divided into two. The earlier half of each data set was used to calibrate the model parameters, with the later halves of the data sets reserved for validation purposes. For the Canberra dataset the calibration data set consisted of records prior to 22:30LT, 12 January 2007, while for the Port Lincoln dataset the calibration dataset consisted of records prior to 00:00LT, 11 January 2005.

The optimal value of $\mu$ was determined using the calibration datasets by minimising the mean differences between the spread index and rates of spread predicted by (5). Figure 1 illustrates how the mean absolute difference and the root mean squared difference between $R_{c g}$ and $S_{\alpha, \mu}$ varies with $\mu$ for the Port Lincoln and Canberra datasets. For the Port Lincoln data the optimal value of $\mu$ is somewhere between 7 and 8 , while for the Canberra data the optimal value is between 4 and 5. The more extreme fire weather conditions (and hence higher rate of spread) in the Port Lincoln dataset results in a larger optimal value of the indraft factor, in accordance with the comments above. 
In light of these results and with the aim of keeping the formulation of the spread index as simple as possible, the indraft factor was taken to have a value of $\mu=6$. Figure 1 indicates that the changes in the mean differences that arise from assuming $\mu=6$ instead of the optimal values of $\mu$ are only relatively minor - they are of the order of $0.03-0.15 \mathrm{~km} \mathrm{~h}^{-1}$.
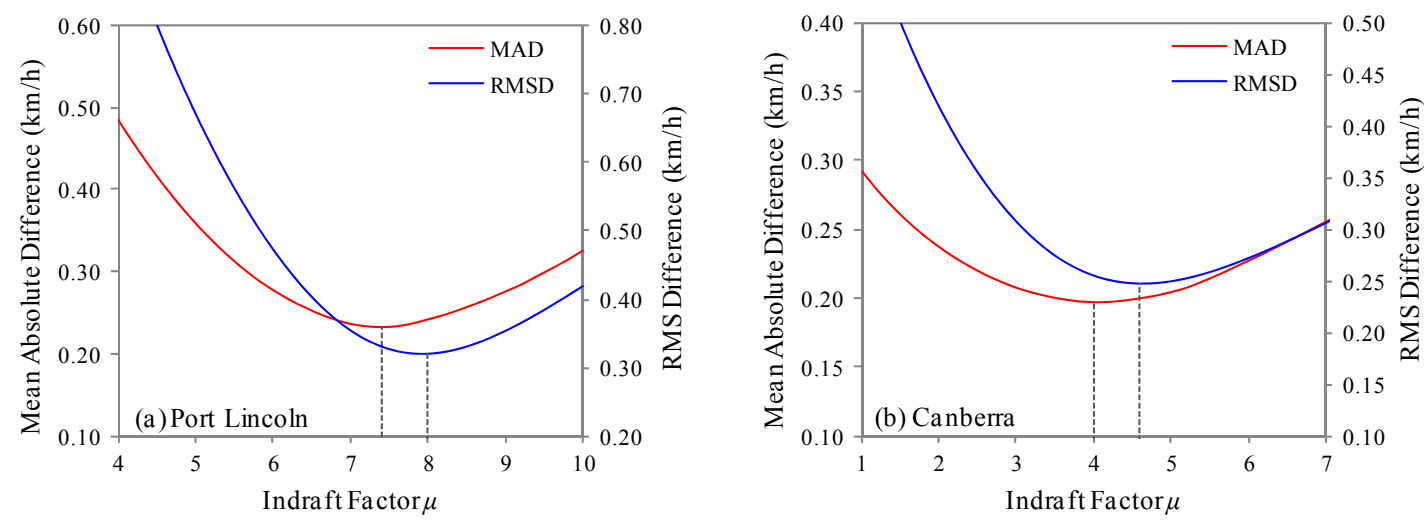

Figure 1. Mean absolute difference (MAD) and root mean squared-difference (RMSD) between $S_{\alpha, \mu}$ and $R_{c g}$ plotted against indraft factor $\mu$ for (a) the Port Lincoln calibration dataset, and (b) the Canberra calibration dataset.

For $\mu=6$, the appropriate value of the scale parameter was found to be $\alpha=2.32$ for the Port Lincoln data, and $\alpha=2.52$ for the Canberra data. As a compromise between these two values, we will take $\alpha=2.4$ for the value of the scale parameter. Our final equation for the spread index $S$ is thus:

$$
S=\frac{2.4 \max (1, U)}{F M I+6}
$$

\section{RESULTS}

To test the efficacy of the spread index we compare the rate of spread predictions delivered by (7) with those predicted by the established grassland fire spread model (5) based on the validation datasets for Port Lincoln and Canberra. In addition, the ability of the spread index to predict rates of spread observed in association with a number of wildfires will also be assessed using data of Cheney et al. (1998, Table 1).

\subsection{Comparison of the spread index with the model of Cheney et al. (1998)}

The Port Lincoln and Canberra validation datasets were used to derive rate of spread predictions using the models (5) and (7). Figure 2 illustrates the results. As can be seen in each case the values of the spread index are highly correlated with the rate of spread predictions derived from the model of Cheney et al. (1998). For the case of the Port Lincoln dataset (top panels in Fig. 2), the values derived from (5) and (7) agree almost exactly for rate of spread values below about $15 \mathrm{~km} \mathrm{~h}^{-1}$. Above $15 \mathrm{~km} \mathrm{~h}^{-1}$ the spread index tends to provide higher estimates of the rate of spread than the model of Cheney et al. (1998). The results based on the Canberra validation dataset (bottom panels in Fig. 2) confirm these findings, though they do illustrate that the spread index produces slightly lower estimates of rate of spread in the range $2 \mathrm{~km} \mathrm{~h}^{-1}-10 \mathrm{~km} \mathrm{~h}^{-1}$.

Summary statistics of the comparative analyses are as follows: for the Canberra validation dataset the linear correlation between rate of spread predictions from (5) and (7) was 0.995 , the rank correlation was 0.993 , the mean absolute difference was $0.210 \mathrm{~km} \mathrm{~h}^{-1}$, and the maximum absolute difference was $0.987 \mathrm{~km} \mathrm{~h}^{-1}$. For the Port Lincoln validation dataset the linear correlation was 0.992, the rank correlation was 0.997, the mean absolute difference was $0.308 \mathrm{~km} \mathrm{~h}^{-1}$, and the maximum absolute difference was $4.27 \mathrm{~km} \mathrm{~h}^{-1}$.

Thus, for both data sets, the rate of spread predictions were very highly correlated and provided rankings of the rate of spread that were very nearly the same. On average the difference between the rate of spread predictions was of the order of $0.2-0.3 \mathrm{~km} \mathrm{~h}^{-1}$, while the maximum differences in rate of spread were less than $1 \mathrm{~km} \mathrm{~h}^{-1}$ for the Canberra dataset and $4.3 \mathrm{~km} \mathrm{~h}^{-1}$ for the Port Lincoln dataset. This maximum difference for the Port Lincoln dataset occurred at the most extreme fire weather conditions experienced during the Wangary fire (wind speed $61 \mathrm{~km} \mathrm{~h}^{-1}$, temperature $42.3^{\circ} \mathrm{C}$ and relative humidity $3 \%$ ). 

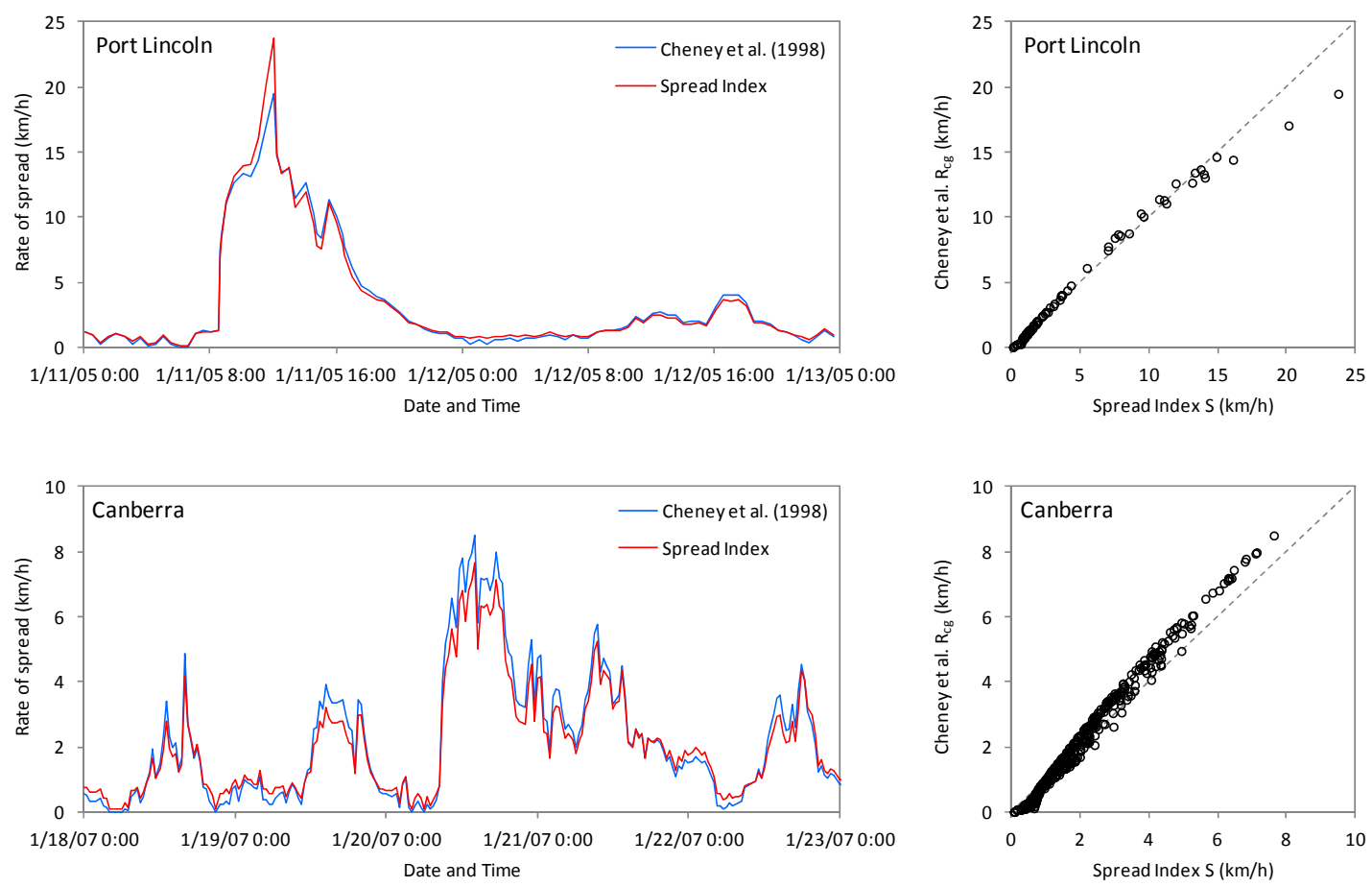

Figure 2. Time series of rate of spread predicted by the spread index (red) and the model of Cheney et al. (blue) as determined from the Port Lincoln validation data set (top left) and the Canberra validation dataset (bottom left). Also shown are scatter plots of rate of spread predictions derived from the model of Cheney et al. against values of the spread index for the Port Lincoln validation data set (top right) and the Canberra validation dataset (bottom right).

\subsection{Comparison of rate of spread predictions with wildfire rate of spread observations}

The wildfire rate of spread observations contained in Cheney et al. (1998, Table 1) are shown plotted against rate of spread predictions derived from (5) and (7) in Figure 3. It is evident that neither the model of Cheney et al. (1998) nor the spread index does a particularly good job of reproducing the estimated rates of spread. The linear correlation between the observations and the predictions of the model of Cheney et al. (1998) was 0.658 , the rank correlation was 0.566 , the mean error was $0.405 \mathrm{~km} \mathrm{~h}^{-1}$, and the maximum absolute error was $8.24 \mathrm{~km} \mathrm{~h}^{-1}$. Comparison of the rate of spread observations with the values of the spread index yielded a linear correlation of 0.566 , a rank correlation of 0.499 , a mean error of $-0.021 \mathrm{~km} \mathrm{~h}^{-1}$, and a maximum absolute error of $9.10 \mathrm{~km} \mathrm{~h}^{-1}$.

\section{DISCUSSION}

A simple index for the rate of spread of a grassland fire was introduced and compared with the CSIRO Grassland Fire Spread model. The so-called spread index can be conceptualized simply as wind speed divided by fuel moisture content, with an adjustment that is consistent with the presence of an intensity dependent indraft wind. It was demonstrated that there is a very tight monotonic relationship between predictions of the spread index and the CSIRO Grassland Fire Spread model, with linear and rank correlations greater than 0.99 .

On average the spread index was able to provide predictions that were within $0.2-0.3 \mathrm{~km} \mathrm{~h}^{-1}$ the rate of spread predictions derived from the CSIRO spread model. The largest differences in predictions offered by the two models were found to occur under conditions of extreme fire weather associated with the 2005 Wangary fire.

Under these conditions the spread index predicts a rate of spread of $23.4 \mathrm{~km} \mathrm{~h}^{-1}$, whereas the CSIRO model predicted a rate of spread of $20.7 \mathrm{~km} \mathrm{~h}^{-1}$ (assuming natural pasture). According to Smith (2005) the Wangary fire spread approximately $21.5 \mathrm{~km}$ between 12:15LT and 13:00LT, which implies an average rate of spread of about $29 \mathrm{~km} \mathrm{~h}^{-1}$. Under these extreme conditions it therefore appears that the spread index provides the more reliable estimate of rate of spread, though no supporting experiments have been conducted under such extreme conditions. 


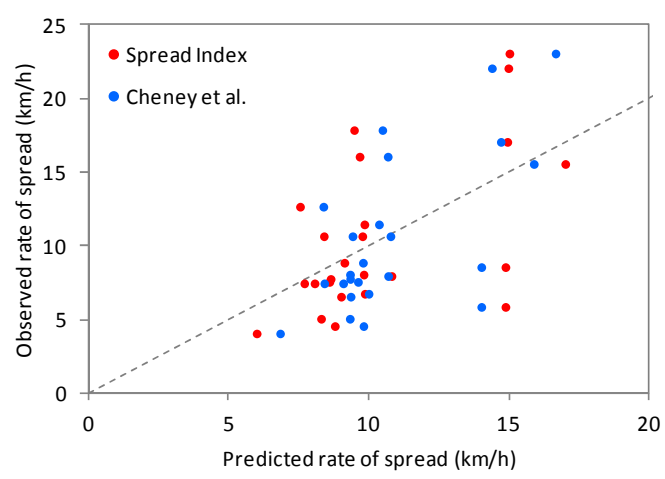

Figure 3. Plots of observed wildfire rate of spread against predicted rates of spread derived from the model of Cheney et al. (1998) (blue dots) and the spread index (red dots).
The form of the spread index shares a lot in common with the CSIRO model, for example: both are products of fuel moisture and wind response functions; fuel moisture is treated as a linear function of temperature and relative humidity; the power law exponent determining the wind speed response in the CSIRO model is 0.844 , which only slightly deviates from the linear response in the spread index; the negative exponential dependence on fuel moisture in the CSIRO model is similar to the truncatedhyperbolic dependence in the spread index. Despite these similarities the spread index depends on only two parameters, whereas the CSIRO spread model has at least twelve parameters that have been fitted using data (double this number again if natural pasture and cut/grazed pasture are treated separately). As such the spread index offers a far more parsimonious model, with no real loss in functionality. The spread index also has obvious pedagogical advantages in terms of its conceptualization.

In work that is as yet unpublished we have found that the spread index also has some promise in predicting rate of spread in other fuel types (eucalypt litter, mallee heath). This suggests that the spread index presented here as a predictor of grassfire rate of spread may have more universal applications.

\section{ACKNOWLEDGMENTS}

J.J. Sharples is supported by a Discovery Indigenous Award IN130100038 of the Australian Research Council and a UNSW Canberra Silver Star Award.

\section{REFERENCES}

Cheney, N.P. and Sullivan, A.L. (1997). Grassfires: fuel, weather and fire behaviour. CSIRO Publishing. Collingwood, Australia.

Cheney, N.P., Gould, J.S. and Catchpole, W.R. (1998). Prediction of fire spread in grasslands. International Journal of Wildland Fire, 8, 1-13.

McArthur, A.G. (1967). Fire behaviour in eucalypt forests. Department of National Development, Forestry and Timber Bureau Leaflet No. 107. Canberra, Australia.

Nelson RM. 2003. Power of the fire - a thermodynamic analysis. International Journal of Wildland Fire 12(1): 51-65.

Pook, E.W., Gill, A.M., 1993. Variation of live and dead fine fuel moisture in Pinus radiata plantations of the Australian Capital Territory. International Journal of Wildland Fire 3 (3), 155-168.

Sharples, J.J., McRae, R.H.D., Weber, R.O. and Gill, A.M. (2009a). A simple index for assessing fuel moisture content. Environmental Modelling and Software, 24, 637-646.

Sharples, J.J., McRae, R.H.D., Weber, R.O. and Gill, A.M. (2009b). A simple index for assessing fire danger rating. Environmental Modelling and Software, 24, 764-774.

Smith, R. (2005). Report of independent review of circumstances surrounding Eyre Peninsula bushfire of $10^{\text {th }}$ and $11^{\text {th }}$ January 2005 (Wangary Bushfire)'. Report commissioned by the South Australian Minister for Emergency Services, May 2005. 95 pp.

Sharples, J.J. and McRae, R.H.D. (2010). Evaluation of a very simple model for predicting the moisture content of eucalypt litter. International Journal of Wildland Fire, 20, 1000-1005.

Slijepcevic, A., Anderson, W.R. and Matthews, S. (2013) Testing existing models for predicting hourly variation in fine fuel moisture in eucalypt forests. Forest Ecology and Management, 306, 202-215.

Sullivan, A.L. (2009). Wildland surface fire spread modelling, 1990-2007. 2: Empirical and quasi-empirical models. International Journal of Wildland Fire, 18, 369-386.

Viney, N.R. (1991). A review of fine fuel moisture modelling. International Journal of Wildland Fire, 1, 215-234. 